\title{
Non-local Graph-based Regularization for Deformable Image Registration
}

\author{
Bartłomiej W. Papież ${ }^{1}$, Adam Szmul ${ }^{1}$, Vicente Grau ${ }^{1}$, \\ Sir J. Michael Brady ${ }^{2}$, and Julia A. Schnabel ${ }^{1,3}$ \\ 1 Institute of Biomedical Engineering, \\ Department of Engineering Science, University of Oxford, UK \\ 2 Department of Oncology, University of Oxford, UK \\ 3 Department of Biomedical Engineering, Division of Imaging Sciences and \\ Biomedical Engineering, King's College London, UK
}

\begin{abstract}
Deformable image registration aims to deliver a plausible spatial transformation between two or more images by solving a highly dimensional, ill-posed optimization problem. Covering the complexity of physiological motion has so far been limited to either generic physical models or local motion regularization models. This paper presents an alternative, graphical regularization model, which captures well the non-local scale of motion, and thus enables to incorporate complex regularization models directly into deformable image registration. In order to build the proposed graph-based regularization, a Minimum Spanning Tree (MST), which represents the underlying tissue physiology in a perceptually meaningful way, is computed first. This is followed by a fast non-local cost aggregation algorithm that performs regularization of the estimated displacement field using the precomputed MST. To demonstrate the advantage of the presented regularization, we embed it into the widely used Demons registration framework. The presented method is shown to improve the accuracy for exhale-inhale CT data pairs.
\end{abstract}

\section{Introduction}

Deformable image registration (DIR) algorithms developed for medical imaging applications generally suffer a trade-off between computational complexity and medical plausibility [1]. From a biomedical standpoint, it is a fundamental requirement that DIR delivers a geometrical transformation that is plausible in an anatomical, physiological, or functional sense, whichever is appropriate to the application. From a mathematical standpoint, DIR is a (very) high-dimensional, ill-posed problem with several millions of degrees of freedom. Many widely-used regularization models are based on a generic physical model such as diffusion [2], while more challenging applications may require additional global constraints, e.g. that the deformation field be a diffeomorphism [3]. Alternatively, in order to accurately model local deformations, a number of local structure or motion preservation models have been proposed [4-6]. However, the deformations within or between human organs are often highly complex and do not occur at a single spatial scale. For example, respiratory-induced motion differs for each lung 
lobe (usually greater in the lower lobes), while the action of the diaphragm and respiratory muscles produces sliding motion at the pleural cavity boundaries. Increasing the plausibility of DIR has to accommodate such complex physiological motions. However, extending the spatial range of the regularization of the deformations to become less local is usually computationally prohibitive.

To overcome these limitations, this paper proposes an alternative, graphbased framework within which such non-local motion constraints, by using a range of spatial scales, can be captured naturally. Previous work in applying graph-based methods to DIR $[7,8]$ has been primarily motivated by the use of efficient discrete optimization for minimizing the data term cost, with less focus on the regularization aspects, which are generally reduced to a fixed scale in a local regularization term. In contrast, here we show how graphical methods enable complex regularization constraints to be incorporated easily and naturally, thus improving on the plausibility of DIR algorithms in general. To introduce our approach in a familiar setting, we show how it can be applied to the classic Thirion's Demons registration $[2,3]$, as an exemplar. Furthermore, the proposed method can easily perform non-local regularization using a minimum spanning tree (MST) derived from a graph representing the desired properties.

This work presents the use of a novel graph-based regularization model for continuous image registration based on Demons as an exemplar $[2,3]$. The motivation for the use of graphs stems from their ability to represent complex connected structures in a perceptually meaningful way. Such structures and connections between them can be represented for example by a MST, which has been shown to replicate well the underlying tissue properties and structure of anatomical connectivity [8]. In contrast to recently proposed anisotropic filter [4], bilateral filter [5], and guided image filter [6] models, the proposed new regularization model implicitly extends local filter kernels to their non-local counterparts by simultaneous consideration of spatial and intensity proximity together with the connectivity of voxel-based nodes. The new MST-based model replaces the Gaussian smoothing originally incorporated in Thirion's Demons $[2,3]$ via employing an efficient MST non-local cost aggregation algorithm [9] to perform regularization on the estimated displacement field. The results achieved by our method on benchmark lung CT data [10] are compared against the best performing Demons [4-6], and a discrete MST optimization framework (deeds) [8].

\section{Methods}

\subsection{Deformable Image Registration}

Deformable image registration (DIR) is generally formulated as the minimization problem of a global energy $\xi$ :

$$
\hat{\boldsymbol{u}}=\arg \min _{\boldsymbol{u}}(\xi(\boldsymbol{u})=\operatorname{Sim}(R, S, \boldsymbol{u})+\alpha \operatorname{Reg}(\boldsymbol{u}))
$$

where the (optimal) displacement field $\hat{\boldsymbol{u}}$ describes the geometrical transformation between two images: the reference image $R$ and the source image $S$. Here, 
Sim measures similarity between $S$ and $R$, while the regularization term Reg encourages the plausibility of the estimated displacement field $\boldsymbol{u}$, and $\alpha \geq 0$ balances the contribution between those two terms. As an example of many, the Demons framework [2] solves the energy given in Eq. (1) in an iterative manner by alternating between minimizing similarity Sim, and regularization Reg, which performs Gaussian smoothing on the estimated displacement field. For a number of medical applications, the Demons algorithm, including the Gaussian regularization, provides very reasonable transformations [3]. However, it has been found less suitable in situations with more complex deformations. Therefore, the anisotropic [4], bilateral-like [5], and guided filter [6] regularization models have been proposed. Although the use of such regularizers considerably improves performance, it remains the case that such approaches perform regularization considering only the predefined local neighborhood around the point of interest, despite the fact that some organs can also deform in a non-local manner. In such cases, neither global nor local regularization models are sufficiently versatile to handle the complexity of the organ motion. To the best of our knowledge, incorporating connections between different anatomical structures has not been considered so far for registration regularization except for the case of registering each presegmented region separately [11].

In the next section, we show how graph-based regularization provides a mathematical framework within which local, semi-local, and global constraints can be accommodated in a natural manner. To introduce the method, we use as an exemplar the widely known and used Demons algorithm [2,3]. However, it is important to note that the method would also apply to other DIR algorithm that can be formulated as an energy minimization as above.

\subsection{Graph-based Regularization for the Demons algorithm}

The overall algorithmic approach may be summarized as follows:

1. calculate the Demons force term (classic step $[2,3]$ )

2. create a graph-based representation for the regularization model

3. find a minimum spanning tree (MST) for this graph

4. perform regularization based on MST via fast tree-cost-aggregation

Step 1 is the classic step, and is described in [2,3]. Steps 2, 3, and 4 form the new contributions of this work and will be described in detail in the following sections.

Step 2: Graph-based representation for regularization. In analogy to [8], we represent the image as a graph, to accommodate the complexity of anatomical structures and connections between them. For this purpose, a graph $G=(V, E)$ is defined comprising a set of nodes $V$ and a set of edges $E$. In this paper, for clarity of exposition, the nodes $p, q \in V$ are assumed to correspond to voxels of the image volume $I_{G}$, and the edges $e \in E$ connect all nearest neighbor nodes (simple 6-connected graph for a $3 \mathrm{D}$ volume) with the edge weight given as the 

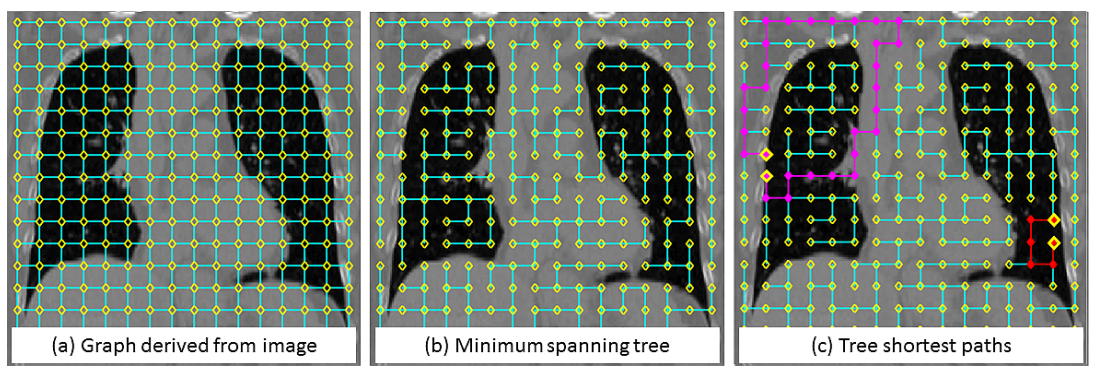

Fig. 1. Visualization of (a) graph derived from image, (b) minimum spanning tree extracted from graph (a), and (c) shortest paths between two nodes in the same (red) and different (magenta) anatomical structures.

absolute difference between the intensity of the node $p$ and the respective voxel in node $q$. However, it has to be understood that the method applies far more generally. An exemplar graph generated for a coronal slice of a lung CT is illustrated in Fig. 1. Although only the nearest neighbor connections are considered, it will be shown later in this work that such connections can extend regularization to a non-local scale in contrast to conventional regularization methods.

Step 3: Finding a minimum spanning tree. To find a minimum spanning tree (MST) for the weighted graph $G$ constructed in the previous section, a greedy Prim-Jarnik (P-J) algorithm is used. The P-J algorithm successively removes any unwanted edges with underlying high intensity differences from the graph, while leaving the edges connecting all nodes in the graph in the form of a tree. In practice, edges with large weights often mark edges crossing different organs, and removing them is thus advantageous in order to avoid regularization across organ boundaries. An exemplar of MST is shown in Fig. 1(b), generated from the graph presented in Fig. 1(a) of a 2D coronal slice of a lung CT. Fig. 1(c) shows the shortest path between different nodes of the graph. As can be seen, the nodes inside the same structure (red) have a short tree distance, whereas nodes within two different structures have a long tree distance despite being spatially close (magenta). Effectively, tree distance is intrinsically more relevant to regularization and deformable registration than is a spatial distance metric such as Euclidean or city block. The tree distance $\delta(p, q)$ in the constructed MST is the sum of lengths of the edges between node $p$ and $q$, where the length of an edge is the Euclidean distance between the two connected nodes (for simplicity the distance between neighbor nodes is defined to be 1).

Step 4: Regularization based on MST via fast tree-cost-aggregation. The final step of incorporating the new graph-based regularization model to the exemplar Demons framework combines a fast guided image filtering procedure [12] with an efficient non-local cost aggregation algorithm for MST [9]. First, a 
minimum spanning tree average operator $\mu^{t}$ (where ${ }^{t}$ refers to tree) of variable $X$ is defined as follows:

$$
\mu_{p}^{t}[X]=\frac{\sum_{q \in V} w(p, q) X(q)}{\sum_{q \in V} w(p, q)}
$$

where $w(p, q)=\exp \left(-\frac{\delta(p, q)}{\sigma}\right)$ denotes a spatial tree similarity (or weight), and $\sigma$ is a weighting parameter. Direct implementation of the MST average operator $\mu^{t}$ (Eq. (2)) is impractical due to the computational cost required to compute the distances between all nodes in the graph. For this reason, Yang [9] proposed an efficient MST cost aggregation algorithm, which requires only two passes through the MST (two additions and three multiplications in total) in order to calculate $\sum_{q \in V} w(p, q) X(q)$. In this paper, we employ this MST cost-aggregation algorithm to perform regularization of the estimated displacement field $\boldsymbol{u}$. In this way, the MST average operator $\mu^{t}$ replaces the standard mean operation in the guided image filter. The guided filter is a local linear model between the guidance image $I_{G}$ and the filter output $\boldsymbol{u}_{\text {out }}$ as in our case we consider displacement field $\boldsymbol{u}_{\text {out }}$ to be the output, and $\boldsymbol{u}_{\text {in }}$ to be the input (we refer a reader to [12] for the detailed derivation of $A$ and $B$ given in Eq. 4). The guided filter is defined as follows [12]:

$$
\boldsymbol{u}_{\text {out }}(p)=\mu_{p}^{t}[A] I_{G}(p)+\mu_{p}^{t}[B]
$$

where:

$$
A(p)=\frac{\mu_{p}^{t}\left[I_{G} \boldsymbol{u}_{i n}\right]-\mu_{p}^{t}\left[I_{G}\right] \mu_{p}^{t}\left[\boldsymbol{u}_{i n}\right]}{\sigma_{p}^{t}\left[I_{G}\right]+\epsilon}, B(p)=\mu_{p}^{t}\left[\boldsymbol{u}_{i n}\right]-A(p) \mu_{p}^{t}\left[I_{G}\right]
$$

$\sigma_{p}^{t}\left[I_{G}\right]=\mu_{p}^{t}\left[I_{G}^{2}\right]-\left(\mu_{p}^{t}\left[I_{G}\right]\right)^{2}$ is a minimum spanning tree variance, and $\epsilon>0$ is a guided filter parameter. Considering the guidance image $I_{G}$, the output displacement field $\boldsymbol{u}_{\text {out }}$ can be filtered with respect to the registered image (selfguidance), or sparse image representation based on supervoxel clustering of the entire thoracic cage [6]. Note that unlike anisotropic [4], guided [6] or bilateral [5] filters, our graph-based method evaluates contributions from all nodes in the graph (volume) to the estimated displacement field during the MST cost aggregation. This substantially improves the regularization model as non-local MST contributions can be captured simply and naturally for the whole structure of interest, whereas the local regularization methods are limited to the (explicit) predefined region of interest only. Although, in theory, we could extend the predefined local patch in [4-6] to cover the full volume (however in practice this would be computationally expensive), here the MST provides an implicit support region without decreasing the overall efficiency.

To visualize the key differences between the local regularization models and the proposed non-local MST method, exemplar kernels for the bilateral model [5], and the proposed non-local MST model are shown in Fig. 2. 


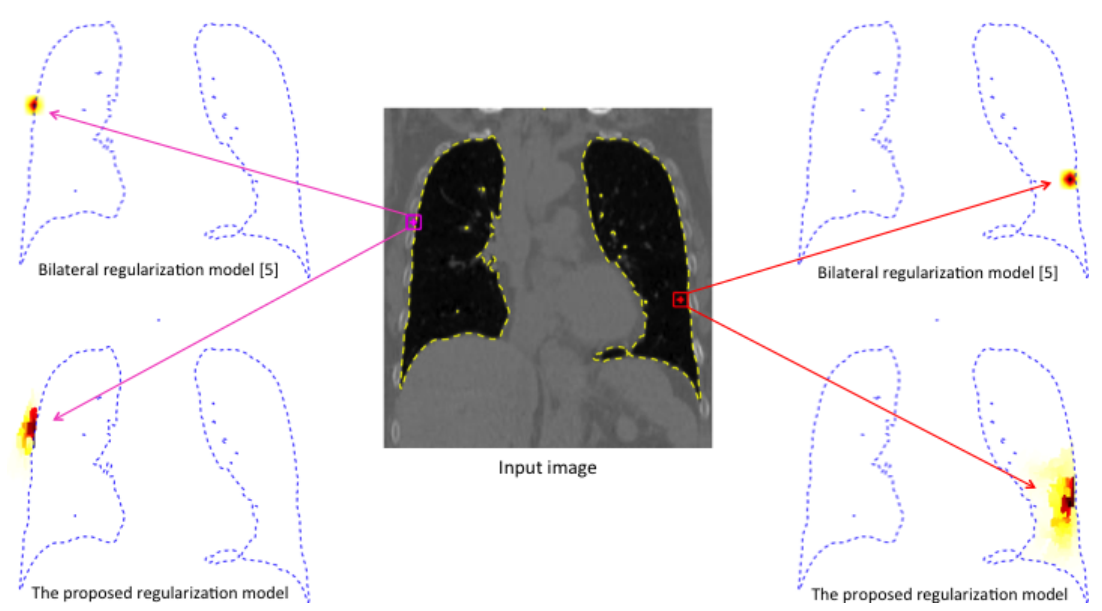

Fig. 2. Comparison between two different kernels based on input image for the local model [5], and the proposed non-local regularization model for two distinctive areas selected inside (red cross) and outside the thoracic cage (magenta cross). The implicit support region for the proposed method can efficiently present the structure of an image contrary to the predefined local region support of [5].

\section{$3 \quad$ Evaluation and Results}

Data. The presented method is evaluated using a publicly available $4 \mathrm{D}$ CT data set [10]. The Dir-Lab data set consists of 10 consecutive respiratory cycle phase volumes with spatial resolution varying between $0.97 \times 0.97 \times 2.5$ and $1.16 \times 1.16 \times 2.5 \mathrm{~mm}^{3}$. To quantify the registration accuracy, the Target Registration Error (TRE) was calculated for the well-distributed set of landmarks, which are provided with this data set (300 landmarks per case for inhale and exhale volumes). In all cases, the end-inhale image was selected as the target image and the end-exhale image as the moving image.

Implementation. For quantitative evaluation of the proposed regularization model, the Demons approach with an update composition scheme and Thirion's symmetric force was implemented (see [3] for details). For simplicity, the proposed method used the Sum of Squared Differences as a similarity measure (also used in $[3-6])$. The following parameters were used for the proposed registration method: $\sigma=2.5$ (Eq. (2)), and $\epsilon=0.1$ (Eq. (4)).

Quantitative Results and Comparison. Table 1 shows the TRE based on 300 well-populated, manually annotated landmarks for all ten cases included in the Dir-Lab data set [10]. The initial TRE is $8.46 \pm 5.5 \mathrm{~mm}$ and the transformations estimated by the proposed method reduce the TRE to $1.44 \pm 1.4 \mathrm{~mm}$, achieving the best result in our comparison. The task of registering these lung 
Table 1. Results achieved by the proposed method (MST) and four other most relevant methods for 3D registration of CT lung from Dir-Lab data set. The proposed method shows the lowest average Target Registration Error (TRE) among all methods.

\begin{tabular}{rrrrlrrl}
\hline & before & dem [3] & bil [5] & \multicolumn{1}{c}{ deeds [8] } & gui $[6]$ & ani $[4]$ & \multicolumn{1}{c}{ MST } \\
\hline c1 & $3.89 \pm 2.9$ & $1.08 \pm 0.6$ & $1.05 \pm 0.5$ & $0.98 \pm 0.5$ & $1.08 \pm 0.5$ & $1.06 \pm 0.6$ & $\mathbf{0 . 8 3} \pm \mathbf{0 . 9}$ \\
c2 & $4.34 \pm 3.9$ & $1.11 \pm 0.6$ & $1.08 \pm 0.6$ & $0.95 \pm 0.5$ & $1.06 \pm 0.5$ & $1.45 \pm 1.0$ & $\mathbf{0 . 8 7} \pm \mathbf{0 . 9}$ \\
c3 & $6.94 \pm 4.1$ & $1.51 \pm 0.9$ & $1.46 \pm 0.9$ & $1.18 \pm 0.7$ & $1.16 \pm 0.6$ & $1.88 \pm 1.4$ & $\mathbf{1 . 1 0} \pm \mathbf{1 . 1}$ \\
c4 & $9.83 \pm 4.9$ & $2.21 \pm 1.8$ & $2.05 \pm 1.5$ & $\mathbf{1 . 6 0} \pm \mathbf{1 . 4}$ & $2.11 \pm 2.2$ & $2.04 \pm 1.4$ & $1.96 \pm 2.0$ \\
c5 & $7.48 \pm 5.5$ & $2.21 \pm 1.9$ & $2.02 \pm 1.7$ & $1.72 \pm 1.5$ & $1.40 \pm 1.2$ & $2.73 \pm 2.1$ & $\mathbf{1 . 3 6} \pm \mathbf{1 . 4}$ \\
c6 & $10.9 \pm 7.0$ & $2.98 \pm 2.6$ & $2.48 \pm 1.8$ & $\mathbf{1 . 7 2} \pm \mathbf{1 . 1}$ & $2.21 \pm 2.6$ & $2.72 \pm 2.0$ & $1.77 \pm 1.6$ \\
c7 & $11.0 \pm 7.4$ & $3.58 \pm 3.5$ & $2.78 \pm 2.3$ & $1.79 \pm 1.4$ & $1.48 \pm 1.1$ & $4.59 \pm 3.4$ & $\mathbf{1 . 5 8} \pm \mathbf{1 . 3}$ \\
c8 & $15.0 \pm 9.0$ & $7.62 \pm 8.1$ & $3.96 \pm 3.8$ & $2.24 \pm 2.6$ & $2.29 \pm 2.7$ & $6.22 \pm 5.7$ & $\mathbf{2 . 0 8} \pm \mathbf{2 . 4}$ \\
c9 & $7.92 \pm 4.0$ & $2.29 \pm 1.7$ & $1.89 \pm 1.2$ & $1.45 \pm 0.8$ & $\mathbf{1 . 3 7} \pm \mathbf{0 . 8}$ & $2.32 \pm 1.4$ & $1.50 \pm 1.2$ \\
$c 10$ & $7.30 \pm 6.4$ & $2.54 \pm 3.1$ & $2.35 \pm 2.5$ & $1.59 \pm 1.6$ & $1.46 \pm 1.3$ & $2.82 \pm 2.5$ & $\mathbf{1 . 4 0} \pm \mathbf{1 . 4}$ \\
\hline$T \bar{R} E$ & $8.46 \pm 5.5$ & $2.71 \pm 1.9$ & $2.11 \pm 0.9$ & $1.52 \pm 1.4$ & $1.56 \pm 1.4$ & $2.78 \pm 2.9$ & $\mathbf{1 . 4 4} \pm \mathbf{1 . 4}$
\end{tabular}

CT data has been also addressed by several methods e.g. deeds with the MSTbased optimization [8] and Demons with guided image filtering [6], which are the most relevant methods for comparison, achieving a higher TRE of $1.52 \pm 1.4 \mathrm{~mm}$, and $1.56 \pm 1.4 \mathrm{~mm}$, respectively. Moreover, the proposed method achieved a significantly lower TRE $(p<0.01)$ when compared to the classic Demons registration, which only achieved a TRE of $2.71 \pm 1.9 \mathrm{~mm}$. Finally, Demons using anisotropic [4] and bilateral filtering [5] obtained a TRE of $2.79 \pm 2.9 \mathrm{~mm}$ and $2.11 \pm 0.9 \mathrm{~mm}$, which is also inferior to the TRE obtained by the presented method. Visualization of the results for the presented method is shown in Fig. 3. Red arrows depict regions of interest where the presented method outperformed the classic Demons.

\section{Discussion and Conclusions}

In this paper, we have presented a new class of regularization model, a graphbased model, that can be easily incorporated into deformable image registration. The new graph-based regularization replicates well complex connected anatomical structures, and therefore improves the accuracy of image registration by implicitly estimating plausible transformations. Furthermore, the use of the efficient minimum spanning tree cost aggregation algorithm extends the predefined local support for regularization to its non-local counterpart. The quantitative evaluation on a publicly available lung CT data set confirmed superiority of the presented registration framework compared to the competing methods in the literature. Future work will include incorporation of prior knowledge combining both structural and functional imaging data coming from PET/CT or PET/MRI system to construct more complex graphs representing underlying characteristics of different organs. 

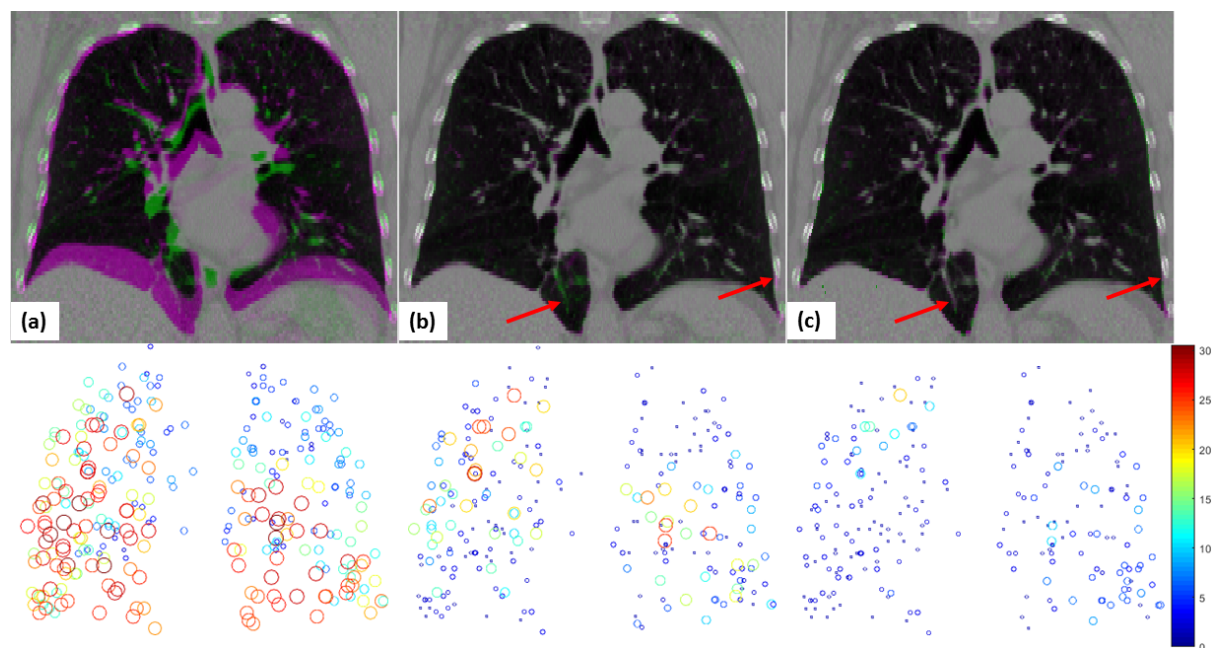

Fig. 3. Visualization of the image intensity differences (top) and $2 \mathrm{D}$ projection of the Target Registration Error (bottom) before registration (a), and after performing (b) classic Demons [3], and (c) the presented method for the most challenging case 8 from Dir-Lab. Color overlay is given for the coronal view of inhale (green) and exhale (magenta) volumes. TRE is projected on the coronal plane and denoted by the size and color of circles. A clear improvement after registration using the presented method is visible.

Acknowledgments. We would like to acknowledge funding from the CRUK/EPSRC Cancer Imaging Centre in Oxford.

\section{References}

1. A. Sotiras, C. Davatzikos, and N. Paragios. Deformable medical image registration: A survey. IEEE Trans. Med. Imaging, 32(7):1153-1190, 2013.

2. J.-P. Thirion. Image matching as a diffusion process: an analogy with Maxwell's demons. Med. Image Anal., 2(3):243-260, Sep 1998.

3. T. Vercauteren, X. Pennec, A. Perchant, and N. Ayache. Diffeomorphic Demons: Efficient non-parametric image registration. NeuroImage, 45:S61-S72, 2009.

4. D.F. Pace, S.R. Aylward, and M. Niethammer. A locally adaptive regularization based on anisotropic diffusion for deformable image registration of sliding organs. IEEE Trans. Med. Imag., 32(11):2114-2126, 2013.

5. B.W. Papież, M.P. Heinrich, J. Fehrenbach, L. Risser, and J.A. Schnabel. An implicit sliding-motion preserving regularisation via bilateral filtering for deformable image registration. Med. Image Anal., 18 (8):1299-1311, 2014.

6. B.W. Papież, J. Franklin, M.P. Heinrich, F.V. Gleeson, and J.A. Schnabel. Liver motion estimation via locally adaptive over-segmentation regularization. In Medical Image Computing Computer-Assisted Intervention, pages 427-434. Springer, 2015.

7. B. Glocker, A. Sotiras, N. Komodakis, and N. Paragios. Deformable medical image registration: setting the state of the art with discrete methods. Annu. Rev. Biomed. Eng., 13:219-244, 2011. 
8. M.P. Heinrich, M Jenkinson, M. Brady, and J.A. Schnabel. MRF-based deformable registration and ventilation estimation of lung CT. IEEE Trans. Med. Imag., 32(7):1239-1248, 2013.

9. Q. Yang. Stereo matching using tree filtering. IEEE Trans. Pattern Anal. Mach. Intell., 37(4):834-846, 2015.

10. R. Castillo, E. Castillo, R. Guerra, V. Johnson, T. McPhail, A. Garg, and T. Guerrero. A framework for evaluation of deformable image registration spatial accuracy using large landmark point sets. Phys. Med. Biol., 54:1849-1870, 2009.

11. Z. Wu, E. Rietzel, V. Boldea, D. Sarrut, and G.C. Sharp. Evaluation of deformable registration of patient lung $4 \mathrm{DCT}$ with subanatomical region segmentations. Med. Phys., 35(2):775-781, Feb 2008.

12. L. Dai, M. Yuan, F. Zhang, and X. Zhang. Fully connected guided image filtering. In Proceedings of the IEEE Int. Conf. on Computer Vision, pages 352-360, 2015. 